\title{
Elastodynamic Response of Thermal Laser Pulse in Micropolar Thermoelastic Mass Diffusion Medium
}

\author{
Rajneesh Kumar ${ }^{1}$ and Arvind Kumar ${ }^{2}$ \\ ${ }^{1}$ Department of Mathematics, Kurukshetra University, Kurukshetra, Haryana 136119, India \\ ${ }^{2}$ Department of Mathematics, Punjab Technical University, Jalandhar, Punjab 144603, India \\ Correspondence should be addressed to Arvind Kumar; arvi.math@gmail.com
}

Received 4 October 2015; Revised 2 December 2015; Accepted 6 December 2015

Academic Editor: Felix Sharipov

Copyright (c) 2016 R. Kumar and A. Kumar. This is an open access article distributed under the Creative Commons Attribution License, which permits unrestricted use, distribution, and reproduction in any medium, provided the original work is properly cited.

\begin{abstract}
The present investigation deals with the deformation in micropolar thermoelastic diffusion medium due to inclined load subjected to thermal laser pulse. Normal mode analysis technique is used to solve the problem. The inclined load is assumed to be a linear combination of a normal load and a tangential load. The closed form expressions of normal stress, tangential stress, couple stress, temperature distribution, and mass concentration are obtained. A computer program has been developed to derive the physical quantities numerically. The variation of normal stress, tangential stress, coupled stress, temperature change, and mass concentration is depicted graphically to show the effect of relaxation times and mass concentration. Some particular cases of interest are deduced from the present investigation.
\end{abstract}

\section{Introduction}

Modern engineering structures are often made up of materials possessing the internal structure. Polycrystalline materials and materials with fibrous or coarse grain structure come in this category. The classical theory of elasticity is not sufficient to explain the phenomenon of high frequency short wave length and ultrasonic waves. So micropolar theory was developed to overcome the shortcomings of the classical theory of elasticity by considering the granular structure of the material of the medium. The micropolar theory of elasticity is applied to materials for problems where the classical theory of elasticity fails owing to the microstructure of the material. The linear theory of micropolar elasticity was developed by Eringen [1]. Under this theory, solids can undergo macrodeformations and microrotations. Also they can support couple stresses in addition to force stresses. Nowacki [2-4] extended the micropolar theory of elasticity to include the thermal effects.

Diffusion is defined as the spontaneous movement of the particles from a high-concentration region to the lowconcentration region, and it occurs in response to a concentration gradient expressed as the change in the concentration due to change in position. Thermal diffusion utilizes the transfer of heat across a thin liquid or gas to accomplish isotope separation. Today, thermal diffusion remains a practical process to separate isotopes of noble gases, for example, Xenon, and other light isotopes, for example, Carbon, for research purposes. In most of the applications, the concentration is calculated using Fick's law. This is a simple law which does not take into consideration the mutual interaction between the introduced substance and the medium into which it is introduced or the effect of temperature of this interaction. However, there is a certain degree of coupling with temperature and temperature gradients as temperature speeds up the diffusion process. The thermodiffusion in elastic solids is due to coupling of fields of temperature and mass diffusion process. The thermodiffusion in elastic solids is due to coupling of fields of temperature, mass diffusion, and of strain in addition to heat and mass exchange with the environment. Nowacki [2-5] developed the theory of thermoelastic diffusion by using coupled thermoelastic model. Dudziak and Kowalski [6], respectively, discussed the theory of thermodiffusion and coupled quasi-stationary problems of thermal diffusion for an elastic layer. They studied the influence of cross-effects arising from the coupling 
of the fields of temperature, mass diffusion, and strain due to which the thermal excitation results in additional mass concentration and generates additional fields of temperature.

Laser technology has a vital application in nondestructive materials testing and evaluation. When a solid is heated with a laser pulse, it absorbs some energy which results in an increase in localized temperature. This causes thermal expansion and generation of the ultrasonic waves in the material. The irradiation of the surface of a solid by pulsed laser light generates wave motion in the solid material. There are generally two mechanisms for such wave generation, depending on the energy density deposited by the laser pulse. At high energy density, a thin surface layer of the solid material melts, followed by an ablation process whereby particles fly off the surface, thus giving rise to forces that generate ultrasonic waves. At low energy density, the surface material does not melt, but it expands at a high rate and wave and wave motion is generated due to thermoelastic processes.

Very rapid thermal processes (e.g., the thermal shock due to exposure to an ultrashort laser pulse) are interesting from the stand point of thermoelasticity, since they require a coupled analysis of the temperature and deformation fields. A thermal shock induces very rapid movement in the structural elements, giving rise to very significant inertial forces and thereby an increase in vibration. Rapidly oscillating contraction and expansion generate temperature changes in materials susceptible to diffusion of heat by conduction [7]. This mechanism has attracted considerable attention due to the extensive use of pulsed laser technologies in material processing and nondestructive testing and characterization $[8,9]$. The so-called ultrashort lasers are those with pulse durations ranging from nanoseconds to femtoseconds. In the case of ultrashort-pulse laser heating, the high intensity energy flux and ultrashort duration lead to very large thermal gradients or ultrahigh heating may exist at the boundaries. In such cases, as pointed out by many investigators, the classical Fourier model, which leads to an infinite propagation speed of the thermal energy, is no longer valid [10]. Researchers have proposed several models to describe the mechanism of heat conduction during short-pulse laser heating, such as the parabolic one-step model [11], the hyperbolic one-step model [12], and the parabolic two-step and hyperbolic two-step models $[13,14]$. It has been found that usually the microscopic two-step models, that is, parabolic and hyperbolic two-step models, are useful for thin films.

Scruby and Drain [15] considered the point source model to study the ultrasonic generation by lasers. They studied the heated surface by laser pulse irradiation in the thermoelastic system as a surface center of expansion (SCOE). They also discussed the applications of laser technology in flaw detection and acoustic microscopy. Rose [16] later presented a more exact mathematical basis. Point source model explains main features of laser-generated ultrasound waves but this model fails to explain precursor in epicenter waves. Later introducing the thermal diffusion McDonald [17] and Spicer et al. [18] proposed a new model known as laser-generated ultrasound model. This model reported excellent agreement between theory and experiment for metal materials. But due to the optical penetration effect, this model cannot be applied to the study of laser-generated ultrasound in nonmetallic material directly. The optical absorption occurs at the surface layer in metallic materials, and the heat penetration is resulting due to heat diffusion. In nonmetallic materials, the laser beam can penetrate the specimen to some finite depth and induced a buried bulk-thermal source, so the features of the laser-generated ultrasound will be significantly different from that in metallic materials.

Dubois et al. [19] experimentally demonstrated that penetration depth plays a very important role in the laserultrasound generation process. Ezzat et al. [20] discussed the thermoelastic behavior in metal films by fractional ultrafast laser. Al-Huniti and Al-Nimr [21] investigated the thermoelastic behavior of a composite slab under a rapid dual-phase lag heating. The comparison of one-dimensional and two-dimensional axisymmetric approaches to the thermomechanical response caused by ultrashort laser heating was studied by Chen et al. [22]. Kim et al. [23] studied thermoelastic stresses in a bonded layer due to pulsed laser radiation. Thermoelastic material response due to laser pulse heating in context of four theorems of thermoelasticity was discussed by Youssef and El-Bary [24]. Theoretical study of the effect of enamel parameters on laser induced surface acoustic waves in human incisor was studied by Yuan et al. [25]. A two-dimensional generalized thermoelastic diffusion problem for a thick plate under the effect of laser pulse thermal heating was studied by Elhagary [26].

In this research, taking into account the mass concentration effect and radiation of ultrashort laser, we have established a model for micropolar thermoelastic medium with mass diffusion. The disturbance due to inclined loads has been studied in the proposed problem. The normal stress, tangential stress, coupled tangential stress, temperature distribution, and mass concentration are obtained numerically.

\section{Basic Equations}

Following Eringen [27], Sherief et al. [28], Kumar and Kansal [29], and Al-Qahtani and Datta [30], the basic equations for homogeneous, isotropic micropolar generalized thermoelastic solid with mass diffusion in the absence of body forces and body couples are given by

$$
\begin{aligned}
& (\lambda+\mu) \nabla(\nabla \cdot \mathbf{u})+(\mu+K) \nabla^{2} \mathbf{u}+K \nabla \times \phi \\
& -\beta_{1}\left(1+\tau_{1} \frac{\partial}{\partial t}\right) \nabla T-\beta_{2}\left(1+\tau^{1} \frac{\partial}{\partial t}\right) \nabla C=\rho \ddot{\mathbf{u}} \\
& \left(\gamma \nabla^{2}-2 K\right) \phi+(\alpha+\beta) \nabla(\nabla \cdot \phi)+K \nabla \times \mathbf{u}=\rho j \ddot{\phi} \\
& K^{*} \nabla^{2} T=\rho c^{*}\left(\frac{\partial}{\partial t}+\tau_{0} \frac{\partial^{2}}{\partial t^{2}}\right) T \\
& +\left(1+\varepsilon \tau_{0} \frac{\partial}{\partial t}\right)\left(\beta_{1} T_{0} \nabla \cdot \dot{\mathbf{u}}-Q\right) \\
& +a T_{0}\left(\frac{\partial}{\partial t}+\gamma_{1} \frac{\partial^{2}}{\partial t^{2}}\right) C
\end{aligned}
$$




$$
\begin{aligned}
& D \beta_{2} \nabla^{2}(\nabla \cdot \mathbf{u})+D a\left(1+\tau_{1} \frac{\partial}{\partial t}\right) \nabla^{2} T \\
& +\left(\frac{\partial}{\partial t}+\varepsilon \tau^{0} \frac{\partial^{2}}{\partial t^{2}}\right) C-D b\left(1+\tau^{1} \frac{\partial}{\partial t}\right) \nabla^{2} C=0, \\
& t_{i j}=\lambda u_{k, k} \delta_{i j}+\mu\left(u_{i, j}+u_{j, i}\right)+K\left(u_{j, i}-\epsilon_{i j k} \phi_{k}\right) \\
& -\beta_{1}\left(1+\tau_{1} \frac{\partial}{\partial t}\right) \delta_{i j} T-\beta_{2}\left(1+\tau^{1} \frac{\partial}{\partial t}\right) \delta_{i j} C, \\
& m_{i j}=\alpha \phi_{r, r} \delta_{i j}+\beta \phi_{i, j}+\gamma \phi_{j, i} .
\end{aligned}
$$

The plate surface is illuminated by laser pulse given by the heat input:

$$
Q=I_{0} f(t) g\left(x_{1}\right) h\left(x_{3}\right)
$$

where $I_{0}$ is the energy absorbed. The temporal profile $f(t)$ is represented as follows:

$$
f(t)=\frac{t}{t_{0}^{2}} e^{-\left(t / t_{0}\right)}
$$

Here $t_{0}$ is the pulse rise time. The pulse is also assumed to have a Gaussian spatial profile in $x_{1}$ :

$$
g(x)=\frac{1}{2 \pi r^{2}} e^{-\left(x_{1}^{2} / r^{2}\right)},
$$

where $r$ is the beam radius and as a function of the depth, $x_{3}$, the heat deposition due to the laser pulse is assumed to decay exponentially within the solid:

$$
h\left(x_{3}\right)=\gamma^{*} e^{-\gamma^{*} x_{3}}
$$

Equation (7) with the aid of (8), (9), and (10) takes the following form:

$$
Q=\frac{I_{0} \gamma^{*}}{2 \pi r^{2} t_{0}^{2}} t e^{-\left(t / t_{0}\right)} e^{-\left(x_{1}^{2} / r^{2}\right)} e^{-\gamma^{*} x_{3}} .
$$

Here $\lambda, \mu, \alpha, \beta, \gamma$, and $K$ are material constants, $\rho$ is mass density, $\mathbf{u}=\left(u_{1}, u_{2}, u_{3}\right)$ is the displacement vector, $\phi=\left(\phi_{1}, \phi_{2}, \phi_{3}\right)$ is the microrotation vector, $T$ is temperature, $T_{0}$ is the reference temperature of the body chosen, $C$ is the concentration of the diffusion material in the elastic body, $K^{*}$ is the coefficient of the thermal conductivity, $c^{*}$ is the specific heat at constant strain, $D$ is the thermoelastic diffusion constant, $a$ is the coefficient describing the measure of thermodiffusion, $b$ is the coefficient describing the measure of mass diffusion effects, $j$ is the microinertia, $\beta_{1}=(3 \lambda+2 \mu+$ $K) \alpha_{t 1}, \beta_{2}=(3 \lambda+2 \mu+K) \alpha_{c 1}, \alpha_{t 1}$ is the coefficient of linear thermal expansion, $\alpha_{c 1}$ is the coefficient of linear diffusion expansion, $t_{i j}$ are components of stress, $m_{i j}$ are components of couple stress, $e_{i j}$ are components of strain, $e_{k k}$ is the dilatation, $\delta_{i j}$ is Kronecker delta function, $\tau^{0}$ and $\tau^{1}$ are the diffusion relaxation times, and $\tau_{0}$ and $\tau_{1}$ are thermal relaxation times with $\tau_{0} \geq \tau_{1} \geq 0$. Here $\tau^{0}=\tau^{1}=\tau_{0}=\tau_{1}=\gamma_{1}=0$ for coupled thermoelastic (CT) theory model. Consider $\tau_{1}=\tau^{1}=0$,

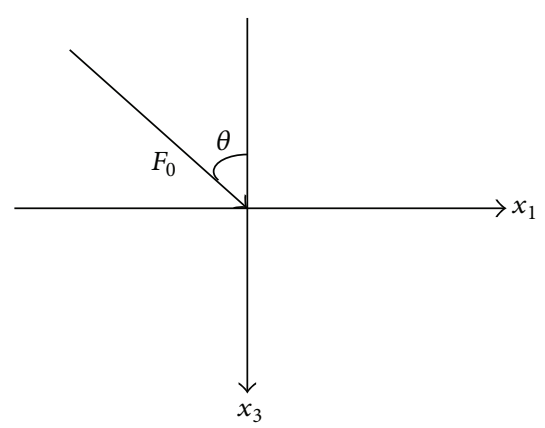

FIGURE 1: Inclined load over a micropolar mass diffusion thermoelastic half-space.

$\epsilon=1, \gamma_{1}=\tau_{0}$ for Lord-Shulman (LS) model and $\varepsilon=0$ and $\gamma_{1}=\tau^{0}$ where $\tau^{0}>0$ for Green-Lindsay (GL) model.

In the above equations symbol ", followed by a suffix denotes differentiation with respect to spatial coordinates and a superposed dot (“.”) denotes the derivative with respect to time, respectively.

\section{Formulation of the Problem}

We consider a micropolar generalized thermoelastic solid with mass diffusion medium with rectangular Cartesian coordinate system $O X_{1} X_{2} X_{3}$ with $x_{3}$-axis pointing vertically downward the medium.

Suppose that an inclined line load $F_{0}$ per unit length is acting on $y$-axis and its inclination to $z$-direction is $\theta$ (Figure 1).

For two-dimensional problems, we take the displacement vector and microrotation vector as

$$
\begin{aligned}
& \mathbf{u}=\left(u_{1}, 0, u_{3}\right), \\
& \phi=\left(0, \phi_{2}, 0\right) .
\end{aligned}
$$

For further consideration it is convenient to introduce in (1)-(5) the dimensionless quantities defined by

$$
\begin{aligned}
u_{i}^{\prime} & =\frac{\rho \omega^{*} c_{1}}{\beta_{1} T_{0}} u_{i}, \\
x_{i}^{\prime} & =\frac{\omega^{*}}{c_{1}} x_{i}, \\
t^{\prime} & =\omega^{*} t, \\
T^{\prime} & =\frac{T}{T_{0}}, \\
\tau_{1}^{\prime} & =\omega^{*} \tau_{1}, \\
\tau_{0}^{\prime} & =\omega^{*} \tau_{0}, \\
\gamma_{1}^{\prime} & =\omega^{*} \gamma_{1}, \\
t_{i j}^{\prime} & =\frac{1}{\beta_{1} T_{0}} t_{i j},
\end{aligned}
$$




$$
\begin{aligned}
& \omega^{*}=\frac{\rho c^{*} c_{1}^{2}}{K^{*}}, \\
& \phi_{i}^{\prime}=\frac{\rho c_{1}^{2}}{\beta_{1} T_{0}} \phi_{i}, \\
& \tau^{1 \prime}=\omega^{*} \tau^{1}, \\
& c_{1}^{2}=\frac{\lambda+2 \mu+k}{\rho}, \\
& c_{2}^{2}=\frac{\mu+k}{\rho}, \\
& c_{3}^{2}=\frac{\gamma}{\rho j}, \\
& c_{4}^{2}=\frac{2 \alpha_{0}}{\rho j_{0}}, \\
& \varepsilon=\frac{\gamma^{2} T_{0}}{\rho^{2} c^{*} c_{1}}, \\
& C^{\prime}=\frac{\beta_{2}}{\rho c_{1}^{2}} C, \\
& c \beta_{1} T_{0} \\
& m_{i j}
\end{aligned}
$$

The displacement components $u_{1}$ and $u_{3}$ are related to the nondimensional potential functions $\phi$ and $\psi$ as follows:

$$
\begin{aligned}
& u_{1}=\frac{\partial \phi}{\partial x_{1}}-\frac{\partial \psi}{\partial x_{3}} \\
& u_{3}=\frac{\partial \phi}{\partial x_{3}}+\frac{\partial \psi}{\partial x_{1}} .
\end{aligned}
$$

Substituting the values of $u_{1}$ and $u_{3}$ from (14) in (1)-(4) with the aid of (12), we obtain

$$
\begin{aligned}
& \nabla^{2} \phi-\ddot{\phi}-\left(1+\tau_{1} \frac{\partial}{\partial t}\right) T-a_{4}\left(1+\tau^{1} \frac{\partial}{\partial t}\right) C=0 \\
& \nabla^{4} \phi+a_{9}\left(1+\tau_{1} \frac{\partial}{\partial t}\right) \nabla^{2} T+a_{10}\left(1+\epsilon \tau^{0} \frac{\partial}{\partial t}\right) \dot{C} \\
& -a_{11}\left(1+\tau^{1} \frac{\partial}{\partial t}\right) \nabla^{2} C=0 \\
& \left(1+\tau_{0} \frac{\partial}{\partial t}\right) \dot{T}+a_{5}\left(\frac{\partial}{\partial t}+\varepsilon \tau_{0} \frac{\partial^{2}}{\partial t^{2}}\right) \nabla^{2} \phi \\
& +a_{8}\left(\frac{\partial}{\partial t}+\gamma_{1} \frac{\partial^{2}}{\partial t^{2}}\right) C \\
& -\nabla^{2} T=Q_{0} f^{*}\left(x_{1}, t\right) e^{-\gamma^{*} x_{3}}
\end{aligned}
$$

$$
\begin{aligned}
& a_{2} \nabla^{2} \psi-\ddot{\psi}+a_{3} \phi_{2}=0, \\
& \nabla^{2} \phi_{2}-2 a_{6} \phi_{2}-a_{6} \nabla^{2} \psi=a_{7} \ddot{\phi}_{2} .
\end{aligned}
$$

Here, $a_{1}=(\lambda+\mu) / \rho c_{1}^{2}, a_{2}=(\mu+K) / \rho c_{1}^{2}, a_{3}=K / \rho c_{1}^{2}, a_{4}=$ $\rho c_{1}^{2} / \beta_{1} T_{0}, a_{5}=\beta_{1}^{2} T_{0} / \rho K^{*} \omega^{*}, a_{6}=K c_{1}^{2} / \gamma \omega^{* 2}, a_{7}=\rho j c_{1}^{2} / \gamma$, $a_{8}=a \rho c_{1}^{4} / \omega^{*} \beta_{2} K^{*}, a_{9}=a \rho c_{1}^{2} / \beta_{1} \beta_{2}, a_{10}=\rho c_{1}^{4} / D \omega^{*} \beta_{2}^{2}$, and $a_{11}=b \rho c_{1}^{2} / \beta_{2}^{2}$.

Here $\nabla^{2}=\partial^{2} / \partial x_{1}^{2}+\partial^{2} / \partial x_{3}^{2}$ is the Laplacian operator

$$
\begin{aligned}
f\left(x_{1}, t\right) & =\left[t+\epsilon \tau_{0}\left(1-\frac{t}{t_{0}}\right)\right] e^{-\left(x_{1}^{2} / r^{2}+t / t_{0}\right)}, \\
Q_{0} & =\frac{a_{13} I_{0} \gamma^{*}}{2 \pi r^{2} t_{0}^{2}} .
\end{aligned}
$$

\section{Solution of the Problem}

The solution of the considered physical variables can be decomposed in terms of the normal modes as in the following form:

$$
\begin{aligned}
& \left\{\phi, \psi, T, \phi_{2}, C\right\}\left(x_{1}, x_{3}, t\right) \\
& \quad=\left\{\bar{\phi}, \bar{\psi}, \bar{T}, \overline{\phi_{2}}, \bar{C}\right\}\left(x_{3}\right) e^{i\left(k x_{1}-\omega t\right)} .
\end{aligned}
$$

Here $\omega$ is the angular velocity and $k$ is wave number.

Making use of (21) and (15)-(19) after some simplifications, we obtain

$$
\begin{aligned}
{\left[\mathbf{D}^{4}+F \mathbf{D}^{2}+G\right] \bar{\psi} } & =0 \\
{\left[A \mathbf{D}^{6}+B \mathbf{D}^{4}+C \mathbf{D}^{2}+E\right] \bar{\phi} } & =f_{1}\left(\gamma^{*}, x_{1}, t\right) e^{-\gamma^{*} x_{3}}, \\
{\left[A \mathbf{D}^{6}+B \mathbf{D}^{4}+C \mathbf{D}^{2}+E\right] \bar{T} } & =f_{2}\left(\gamma^{*}, x_{1}, t\right) e^{-\gamma^{*} x_{3}}, \\
{\left[A \mathbf{D}^{6}+B \mathbf{D}^{4}+C \mathbf{D}^{2}+E\right] \bar{C} } & =f_{3}\left(\gamma^{*}, x_{1}, t\right) e^{-\gamma^{*} x_{3}},
\end{aligned}
$$

where $\mathbf{D}=d / d x_{3}, A=-a_{21}, B=\left(a_{17} a_{18}+k_{2} a_{21}-a_{22}-\right.$ $\left.a_{18} a_{19}\right), C=\left[a_{17}\left(a_{20}-k^{2} a_{19}\right)-a_{23}+k_{2} a_{22}+a_{18} a_{25}\right], E=$ $\left(-k^{2} a_{20} a_{17}+k_{2} a_{23}-a_{18} a_{26}\right), F=\left(\left(a_{3} a_{6}-a_{2} k_{4}-k_{3}\right) / a_{2}\right)$, $G=\left(\left(k_{3} k_{4}-a_{3} a_{6} k^{2}\right) / a_{2}\right), k_{1}=k^{2}-\omega^{2}, k_{2}=k^{2}-\left(\iota \omega+\tau_{0} \omega^{2}\right)$, $k_{3}=\left(a_{2} k^{2}-\omega^{2}\right), k_{4}=k^{2}+2 a_{6}-\omega^{2} a_{7}, a_{12}=\left(1-\imath \omega \tau_{1}\right)$, $a_{13}=a_{4}\left(1-\iota \omega \tau^{1}\right), a_{14}=a_{9}\left(1-\iota \omega \tau_{1}\right), a_{15}=a_{10}\left(1-\iota \omega \varepsilon \tau^{0}\right)$, $a_{16}=a_{11}\left(1-\iota \omega \tau^{1}\right), a_{17}=-a_{5}\left(\iota \omega+\varepsilon \tau_{0} \omega^{2}\right), a_{18}=a_{8}\left(\iota \omega+\gamma_{1} \omega^{2}\right)$, $a_{19}=a_{12} a_{16}+a_{13} a_{14}, a_{21}=a_{16}-a_{13}, a_{20}=-\left(a_{12}\left(a_{15}+a_{16} k^{2}\right)+\right.$ $\left.a_{13} a_{14} k^{2}\right), a_{22}=\left(2 a_{13} k^{2}-a_{15}-\left(k^{2}+k_{1}\right) a_{16}\right), a_{24}=a_{12}+a_{14}$, $a_{23}=\left(k_{1} a_{15}+k_{1} a_{16} k^{2}-a_{13} k^{4}\right), a_{25}=-\left(a_{14}\left(k_{1}+k^{2}\right)+2 a_{12} k^{2}\right)$, and $a_{26}=\left(a_{14} k_{1} k^{2}+a_{12} k^{4}\right)$. 
The solution of the above system of (22)-(25) satisfying the radiation conditions that $\left(\bar{\phi}, \bar{\psi}, \bar{T}, \overline{\phi_{2}}, \bar{C}\right) \rightarrow 0$ as $x_{3} \rightarrow$ $\infty$ is given as follows:

$$
\begin{aligned}
(\bar{\phi}) & =\sum_{i=1}^{3} c_{i} e^{-m_{i} x_{3}}+\frac{f_{1}}{f_{4}} e^{-\gamma^{*} x_{3}}, \\
\bar{T} & =\sum_{i=1}^{3} \alpha_{i} c_{i} e^{-m_{i} x_{3}}+\frac{f_{2}}{f_{4}} e^{-\gamma^{*} x_{3}},
\end{aligned}
$$

$$
\begin{aligned}
\bar{C} & =\sum_{i=1}^{3} \beta_{i} c_{i} e^{-m_{i} x_{3}}+\frac{f_{3}}{f_{4}} e^{-\gamma^{*} x_{3}}, \\
\left(\bar{\psi}, \overline{\phi_{2}}\right) & =\sum_{i=4}^{5}\left(1, \delta_{i}\right) c_{i} e^{-m_{i} x_{3}},
\end{aligned}
$$

where $m_{i}^{2}(i=1,2,3)$ are the roots of $(22)$ and $m_{i}^{2}(i=4,5)$ are the roots of characteristic equation of (25) and

$$
\begin{aligned}
\alpha_{i} & =-\frac{b_{4}\left(m_{i}^{2}-k_{1}\right)-b_{5}\left(m_{i}^{2}-k_{1}\right)\left(m_{i}^{2}-k^{2}\right)+b_{2}\left(m_{i}^{4}+k^{4}-2 m_{i}^{2} k^{2}\right)}{-b_{1} b_{4}+b_{1} b_{5}\left(m_{i}^{2}-k^{2}\right)+b_{2} b_{3}\left(m_{i}^{2}-k^{2}\right)}, \\
\beta_{i} & =\frac{b_{3}\left(m_{i}^{2}-k_{1}\right)\left(m_{i}^{2}-k^{2}\right)+b_{1}\left(m_{i}^{4}+k^{4}-2 m_{i}^{2} k^{2}\right)}{-b_{1} b_{4}+b_{1} b_{5}\left(m_{i}^{2}-k^{2}\right)+b_{2} b_{3}\left(m_{i}^{2}-k^{2}\right)}, \quad i=1,2,3, \\
\delta_{i} & =\frac{\left(m_{i}^{2}-k_{4}\right)}{a_{6}\left(m_{i}^{2}-k^{2}\right)}, \quad i=4,5, \\
f_{1} & =a_{19} \gamma^{* 2}+a_{20}, \\
f_{2} & =a_{21} \gamma^{* 4}+a_{22} \gamma^{* 2}+a_{23}, \\
f_{3} & =a_{24} \gamma^{* 4}+a_{25} \gamma^{* 2}+a_{26}, \\
f_{4} & =A \gamma^{* 6}+B \gamma^{* 4}+C \gamma^{* 2}+E, \\
f_{i}\left(\gamma^{*}, x_{1}, t\right) & =Q_{0} f\left(x_{1}, t\right) f_{i}, \quad i=1,2,3 .
\end{aligned}
$$

Substituting the values of $\bar{\phi}, \bar{T}, \bar{\psi}, \bar{\phi}_{2}$, and $\bar{C}$ from (26) in (5)-(6) and using (12)-(13) and (21) and solving the resulting equations, we obtain

$$
\begin{gathered}
\bar{t}_{33}=\sum_{i=1}^{5} G_{1 i} e^{-m_{i} x_{3}}-M_{1} e^{-\gamma^{*} x_{3}}, \quad i=1,2, \ldots, 5, \\
\bar{t}_{31}=\sum_{i=1}^{5} G_{2 i} e^{-m_{i} x_{3}}-M_{2} e^{-\gamma^{*} x_{3}}, \quad i=1,2, \ldots, 5, \\
\bar{m}_{32}=\sum_{i=1}^{5} G_{3 i} e^{-m_{i} x_{3}}-M_{3} e^{-\gamma^{*} x_{3}}, \quad i=1,2, \ldots, 5, \\
\bar{T}=\sum_{i=1}^{5} G_{4 i} e^{-m_{i} x_{3}}-M_{4} e^{-\gamma^{*} x_{3}}, \quad i=1,2, \ldots, 5, \\
\bar{C}=\sum_{i=1}^{5} G_{5 i} e^{-m_{i} x_{3}}-M_{5} e^{-\gamma^{*} x_{3}}, \quad i=1,2, \ldots, 5 .
\end{gathered}
$$

Consider $G_{m i}=g_{m i} C_{i}, i=1,2, \ldots, 5$.

Here, $g_{1 i}=b_{2}\left(m_{i}^{2}-k^{2}\right)+b_{3} m_{i}^{2}-\left(1-\imath \omega \tau_{1}\right) \alpha_{i}-b_{11} \beta_{1}(1-$ $\left.\iota \omega \tau^{1}\right), g_{2 i}=-\imath b_{5} k m_{i}, g_{3 i}=0, g_{4 i}=m_{i} \alpha_{i}, g_{5 i}=m_{i} \beta_{i}$, $i=1,2,3$, and $g_{1 l}=-\imath k b_{3} m_{l} \delta_{l}, g_{2 l}=\left(b_{6} m_{l}^{2}+b_{5} k^{2}\right) \delta_{l}-b_{7}$, $g_{3 l}=b_{8} m_{l}, g_{4 l}=0, g_{5 l}=0, l=4,5, M_{1}=\left(\left(b_{11} f_{3}\left(1-\iota \omega \tau^{1}\right)-\right.\right.$ $\left.\left.b_{2} f_{1}\left(\gamma^{* 2}-k^{2}\right)-b_{3} f_{1}+\tau_{11} f_{2}\right) / f_{4}\right), M_{2}=-\iota b_{5} k \gamma^{*} f_{1} / f_{4}, M_{3}=0$, $M_{4}=-\gamma^{*} f_{2} / f_{4}$, and $M_{5}=-\gamma^{*} f_{3} / f_{4}$.

\section{Boundary Conditions}

We consider normal and tangential force acting at the surface $x_{3}=0$ along with vanishing of couple stress in addition to thermal and mass concentration boundaries considered at $x_{3}=0$ and for $I_{0}=0$. Mathematically, this can be written as

$$
\begin{aligned}
t_{33} & =-F_{1} e^{-i\left(k x_{1}-\omega t\right)}, \\
t_{31} & =-F_{2} e^{-i\left(k x_{1}-\omega t\right)}, \\
m_{32} & =0, \\
h_{1} \frac{\partial T}{\partial x_{3}}+h_{2} T & =0, \\
h_{3} \frac{\partial C}{\partial x_{3}}+h_{4} C & =0,
\end{aligned}
$$

where $F_{1}$ and $F_{2}$ are the magnitude of the applied force. 
Here, $h_{2}, h_{4} \rightarrow 0$ corresponds to insulated impermeable boundaries. Similarly, $h_{1}, h_{3} \rightarrow 0$ corresponds to isothermal and isoconcentrated boundaries.

Substituting the expression of the variables considered into these boundary conditions, we can obtain the following equations:

$$
\begin{aligned}
& \sum_{i=1}^{5} g_{1 i} c_{i}=-F_{1}, \\
& \sum_{i=1}^{5} g_{2 i} c_{i}=-F_{2},
\end{aligned}
$$

$$
\sum_{i=1}^{5} g_{3 i} c_{i}=0
$$$$
\sum_{i=1}^{5}\left(h_{2}-m_{i} h_{1}\right) g_{1 i} c_{i}=0
$$$$
\sum_{i=1}^{5}\left(h_{4}-m_{i} h_{3}\right) g_{1 i} c_{i}=0
$$

Equations (41) are solved by using the matrix method as follows:

$$
\left[\begin{array}{l}
c_{1} \\
c_{2} \\
c_{3} \\
c_{4} \\
c_{5}
\end{array}\right]=\left[\begin{array}{ccccc}
g_{11} & g_{12} & g_{13} & g_{14} & g_{15} \\
g_{21} & g_{22} & g_{23} & g_{24} & g_{25} \\
g_{31} & g_{32} & g_{33} & g_{34} & g_{35} \\
\left(h_{2}-m_{1} h_{1}\right) g_{41} & \left(h_{2}-m_{2} h_{1}\right) g_{42} & \left(h_{2}-m_{3} h_{1}\right) g_{43} & \left(h_{2}-m_{4} h_{1}\right) g_{44} & \left(h_{2}-m_{5} h_{1}\right) g_{45} \\
\left(h_{4}-m_{1} h_{3}\right) g_{51} & \left(h_{4}-m_{2} h_{3}\right) g_{52} & \left(h_{4}-m_{3} h_{3}\right) g_{53} & \left(h_{4}-m_{4} h_{3}\right) g_{54} & \left(h_{4}-m_{5} h_{3}\right) g_{55}
\end{array}\right]^{-1}\left[\begin{array}{c}
-F_{1} \\
-F_{2} \\
0 \\
0 \\
0
\end{array}\right] .
$$

5.1. Applications. Inclined Line Load. For an inclined line load $F_{0}$ we have

$$
\begin{aligned}
& F_{1}=F_{0} \cos \theta, \\
& F_{2}=F_{0} \sin \theta .
\end{aligned}
$$

Making use of (42) in (41), we obtain the corresponding expressions of normal stress, tangential stress, couple stress, temperature distribution, and mass concentration due to inclined load.

\subsection{Particular Cases}

(i) If we take $\tau_{1}=\tau^{1}=0$ and $\varepsilon=1$, in (41) with the aid of (43), we obtain the corresponding expressions of stresses, displacements, and temperature distribution for micropolar mass diffusion thermoelastic halfspace with one relaxation time.

(ii) If we take $\varepsilon=0$ in (41) with the aid of (43), the corresponding expressions of stresses, displacements, and temperature distribution are obtained for micropolar mass diffusion thermoelastic half-space with two relaxation times.

(iii) Taking $\tau^{0}=\tau^{1}=\tau_{0}=\tau_{1}=0$ in (41) with the aid of (43) yields the corresponding expressions of stresses, displacements, and temperature distribution for micropolar mass diffusion coupled thermoelastic half-space.

(iv) This is the case where $\theta=0$ corresponds to normal load.

(v) This is the case where $\theta=\pi / 2$ corresponds to tangential load.

\subsection{Special Case}

Micropolar Thermoelastic Medium. If we neglect the diffusion effect in (41) with the aid of (43), we obtain the corresponding expressions of stresses, displacements, and temperature for micropolar generalized thermoelastic half-space.

\section{Numerical Results and Discussions}

The analysis is conducted for a magnesium crystal-like material. For numerical computations, following Eringen [31], the values of physical constants are as follows:

$$
\begin{aligned}
& \lambda=9.4 \times 10^{10} \mathrm{Nm}^{-2}, \mu=4.0 \times 10^{10} \mathrm{Nm}^{-2}, K=1.0 \times \\
& 10^{16} \mathrm{Nm}^{-2}, \rho=1.74 \times 10^{3} \mathrm{Kgm}^{-3}, j=0.2 \times 10^{-19} \mathrm{~m}^{2}, \\
& \gamma=0.779 \times 10^{-9} \mathrm{~N} .
\end{aligned}
$$

Following Dhaliwal and Singh [32], thermal and diffusion parameters are given by

$$
\begin{aligned}
& c^{*}=1.04 \times 10^{3} \mathrm{JKg}^{-1} \mathrm{~K}^{-1}, K^{*}=1.7 \times 10^{6} \mathrm{Jm}^{-1} \mathrm{~s}^{-1} \mathrm{~K}^{-1}, \\
& \alpha_{t 1}=2.33 \times 10^{-5} \mathrm{~K}^{-1}, \alpha_{c 1}=2.48 \times 10^{10} \mathrm{~K}^{-1}, T_{0}= \\
& 298 \mathrm{~K}, \tau_{0}=0.02, \tau_{1}=0.01, \alpha_{c 1}=2.65 \times 10^{-4} \mathrm{~m}^{3} \mathrm{Kg}^{-1}, \\
& a=2.9 \times 10^{4} \mathrm{~m}^{2} \mathrm{~s}^{-2} \mathrm{~K}^{-1}, b=32 \times 10^{5} \mathrm{Kg}^{-1} \mathrm{~m}^{5} \mathrm{~s}^{-2}, \\
& \tau^{1}=0.04, \tau^{0}=0.03, D=0.85 \times 10^{-8} \mathrm{Kgm}^{-3} \mathrm{~s} .
\end{aligned}
$$

A comparison of the dimensionless form of the field variables for the cases of micropolar thermoelastic mass diffusion medium with a laser pulse (MPL) and micropolar thermoelastic mass diffusion medium without a laser pulse (MP), for two different values of inclined angle $\theta(\theta=0$ and $\theta=\pi / 2$ ), is presented in Figures 2-6. The values of all physical quantities for all cases are shown in the range $0 \leq x_{3} \leq 2$.

Solid lines and dash lines correspond to micropolar thermoelastic mass diffusion medium with a laser pulse 


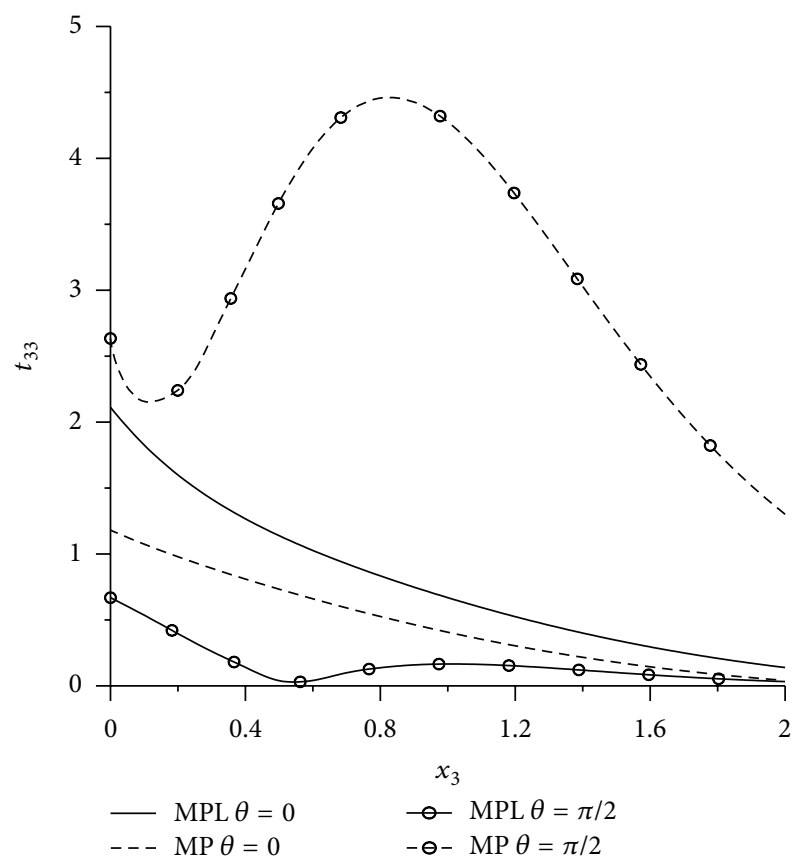

FIGURE 2: Variation of normal stress with respect to distance $x_{3}$.

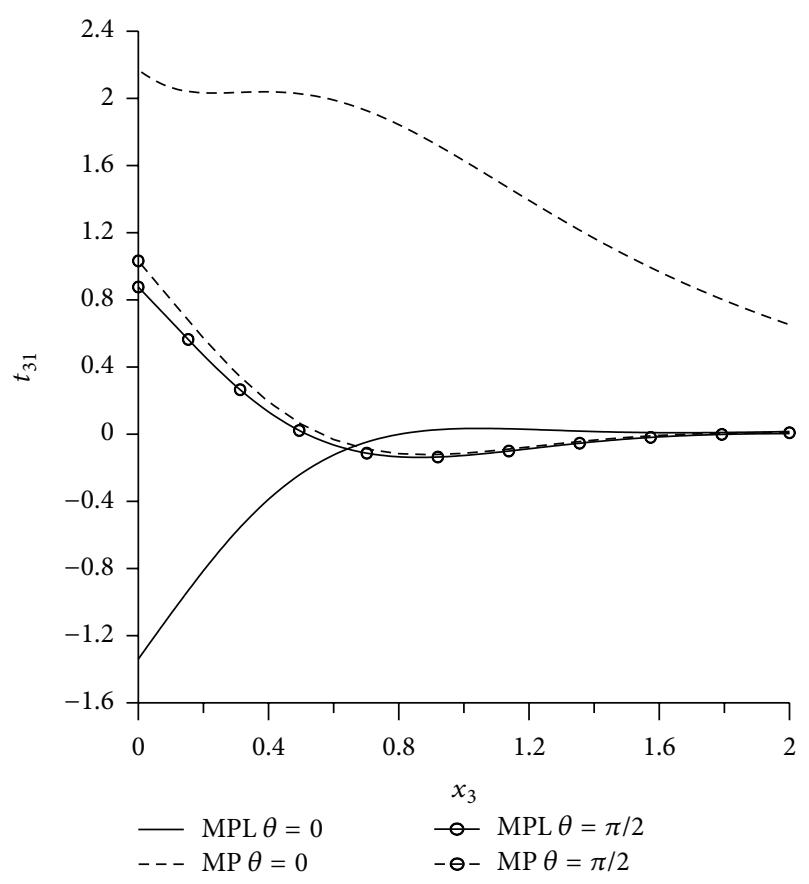

FIGURE 3: Variation of tangential stress with respect to distance $x_{3}$.

(MPL) and micropolar thermoelastic mass diffusion medium without a laser pulse (MP), respectively, for $\theta=0$.

Solid lines with central symbol and dash lines with central symbol correspond to micropolar thermoelastic mass diffusion medium with a laser pulse (MPL) and micropolar thermoelastic mass diffusion medium without a laser pulse (MP), respectively, for $\theta=\pi / 2$.

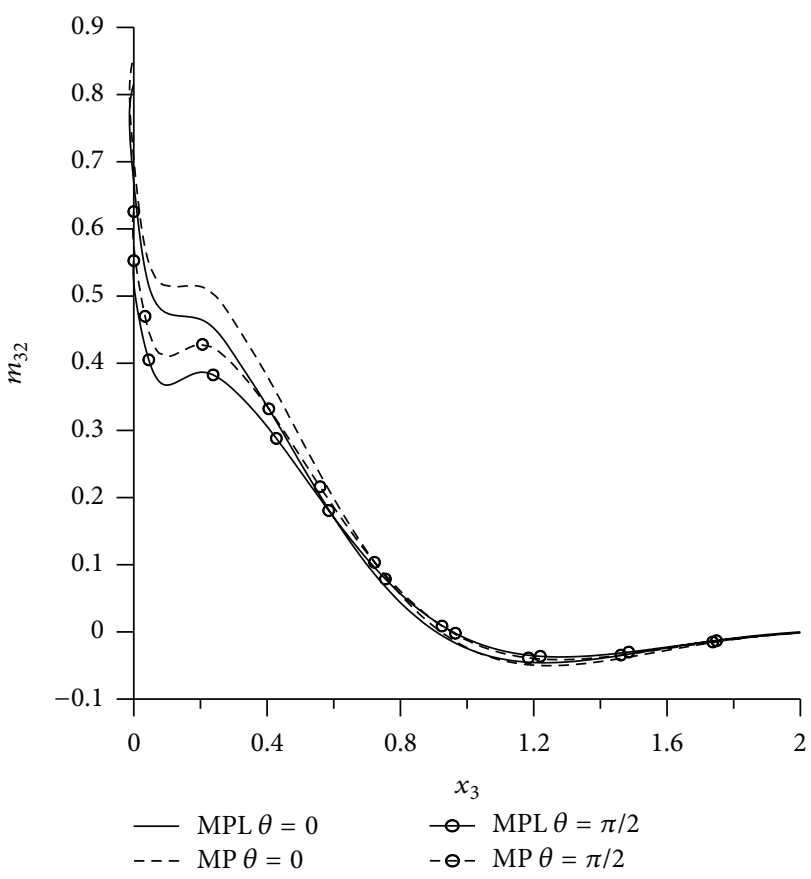

Figure 4: Variation of coupled tangential stress with respect to $x_{3}$.

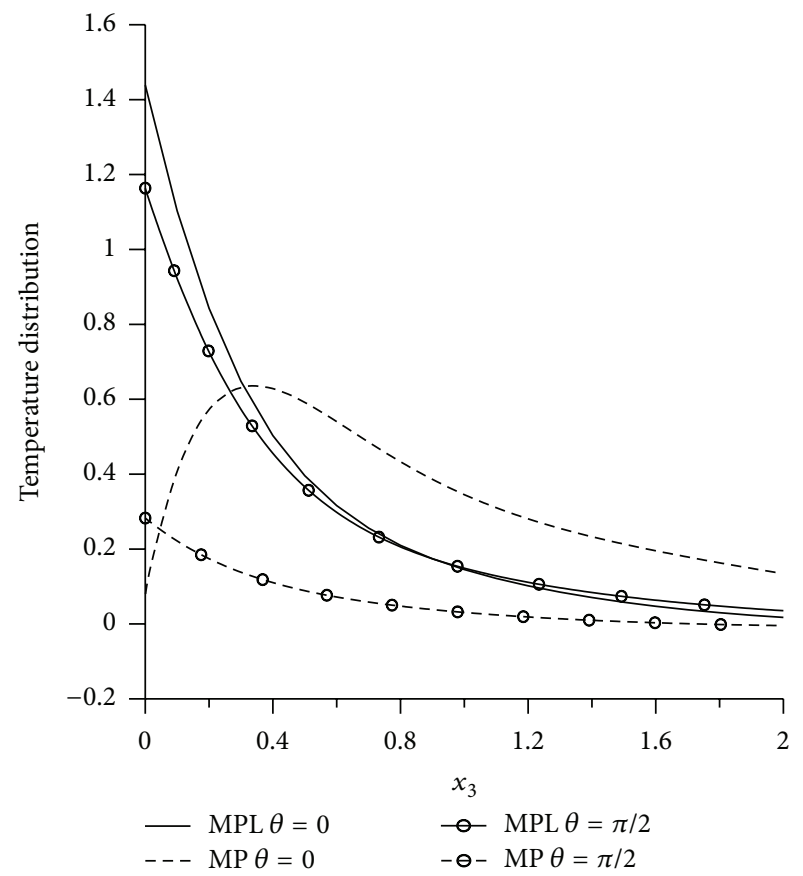

FIgURE 5: Variation of temperature distribution with respect to $x_{3}$.

The computations were carried out in the absence and presence of laser pulse $\left(I_{0}=10^{5}, 0\right)$ and, on the surface of plane $x_{1}=1, t=0.1$.

6.1. Insulated Impermeable Boundary. Figure 2 shows the variations of normal stress component $t_{33}$ with $x_{3}$. For both two values of inclination angle $t_{33}$ shows similar behavior. The normal stress has maximum value near the application of 


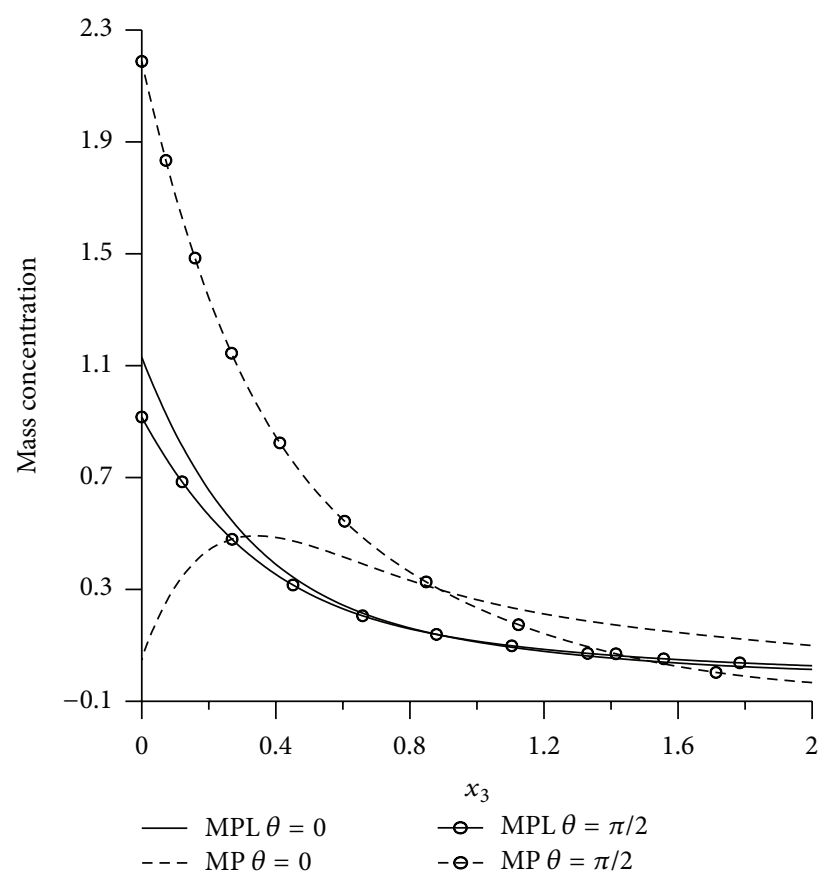

FIGURE 6: Variation of mass concentration with respect to $x_{3}$.

source. Initially the value of normal stress $t_{33}$ decreases as $x_{3}$ increases. The angle of inclination effect on the normal stress can be seen in this figure. For $\theta=0$ the normal stress initially decreases and then increases and thereafter approaches the boundary surface away from the application of normal force.

Figure 3 shows the variation of tangential stress $t_{31}$ with the distance $x_{3}$. We notice that initially $t_{31}$ shows similar behavior for $\theta=0$ and $\theta=\pi / 2$ for MP. The initial behaviors of $t_{31}$ in case of MPL for $\theta=0$ and $\theta=\pi / 2$ is opposite to each other due to the difference between the angles of inclination of load applied. Away from the load applied the value of $t_{31}$ approaches boundary surface.

Figure 4 displays the variation of coupled tangential stress $m_{32}$ with the distance $x_{3}$. It is noticed that initially the behavior of $m_{32}$ for all values of angle of inclination is similar. Also it is observed that the variation in coupled tangential stress for $\theta=0$ is comparable to the variation for $\theta=\pi / 2$. The values of $m_{32}$ for both values of $\theta$ remain oscillatory and approach the boundary surface away from the applied force.

Figure 5 displays the variation of temperature $T$ with distance $x_{3}$. The values of temperature $T$ attain the maximum value near the application of source and away from it decreases and approaches the boundary surface for all the angles of inclination. In case of micropolar thermoelastic medium without laser pulse for $\theta=0$ the temperature initially shows increasing trend but later on this trend is similar to other curves.

Figure 6 displays the variation of mass concentration $C$ with distance $x_{3}$. The value of $C$ decreases near the application of the source and then approaches the boundary surface far away from the load applied.

\section{Conclusion}

The problem of laser irradiation on micropolar thermoelastic mass diffusion medium is a significant problem in continuum mechanics. It is observed that the physical quantities are also affected by the different nonclassical theories of thermoelasticity with mass diffusion. It is observed from Figures 2-6 that angle of inclination effects on stress components, temperature change, and mass concentration depend upon the distance $x_{3}$.

The present problem has a significant application in geophysics and electronics engineering. The effect of diffusion is used to improve the conditions of oil extractions (seeking ways of more efficiently recovering oil from oil deposits). Also the study of thermal and diffusion effects plays an important role in understanding many seismological processes. Nowadays, there is a great deal of interest in the study of this phenomenon due to its application in geophysics and electronic industry.

The results obtained here are useful in engineering problems particularly in the determination state of stresses in a micropolar thermoelastic mass diffusion medium. Also, any particular case of special interest can be derived by assigning suitable values to the parameters and functions in the problem.

\section{Conflict of Interests}

The authors declare that there is no conflict of interests regarding the publication of this paper.

\section{References}

[1] A. C. Eringen, "Linear theory of micropolar elasticity," Indiana University Mathematics Journal, vol. 15, no. 6, pp. 909-923, 1966.

[2] W. Nowacki, "Dynamical problems of thermo diffusion in solids I," Bulletin of the Polish Academy of Sciences: Technical Sciences, vol. 22, pp. 55-64, 1974.

[3] W. Nowacki, "Dynamical problems of thermo diffusion in solids II," Bulletin of The Polish Academy of Sciences: Technical Sciences, vol. 22, pp. 129-135, 1974.

[4] W. Nowacki, "Dynamical problems of thermo diffusion in solids III," Bulletin of the Polish Academy of Sciences: Technical Sciences, vol. 22, pp. 257-266, 1974.

[5] W. Nowacki, "Dynamic problems of diffusion in solids," Engineering Fracture Mechanics, vol. 8, no. 1, pp. 261-266, 1976.

[6] W. Dudziak and S. J. Kowalski, "Theory of thermodiffusion for solids," International Journal of Heat and Mass Transfer, vol. 32, no. 11, pp. 2005-2013, 1989.

[7] D. Trajkovski and R. Čukić, "A coupled problem of thermoelastic vibrations of a circular plate with exact boundary conditions," Mechanics Research Communications, vol. 26, no. 2, pp. 217-224, 1999.

[8] X. Wang and X. Xu, "Thermoelastic wave induced by pulsed laser heating," Applied Physics A, vol. 73, no. 1, pp. 107-114, 2001.

[9] X. Wang and X. Xu, "Thermoelastic wave in metal induced by ultrafast laser pulses," Journal of Thermal Stresses, vol. 25, no. 5, pp. 457-473, 2002.

[10] D. D. Joseph and L. Preziosi, "Heat waves," Reviews of Modern Physics, vol. 61, no. 1, pp. 41-73, 1989. 
[11] M. N. Ozisik and D. Y. Tzou, "On the wave theory in heatconduction," Journal of Heat Transfer, vol. 116, no. 3, pp. 526$535,1994$.

[12] W. S. Kim, L. G. Hector Jr., and M. N. Özisik, "Hyperbolic heat conduction due to axisymmetric continuous or pulsed surface heat sources," Journal of Applied Physics, vol. 68, no. 11, pp. 54785485, 1990.

[13] T. Q. Qiu and C. L. Tien, "Heat transfer mechanisms during short-pulse laser heating of metals," Journal of Heat Transfer, vol. 115, no. 4, pp. 835-841, 1993.

[14] D. Y. Tzou, Macro-To Micro-Scale Heat Transfer: The Lagging Behavior, Taylor \& Francis, Bristol, UK, 1997.

[15] C. B. Scruby and L. E. Drain, Laser Ultrasonics Techniques and Applications, Adam Hilger, Bristol, UK, 1990.

[16] L. R. F. Rose, "Point-source representation for laser-generated ultrasound," Journal of the Acoustical Society of America, vol. 75, no. 3, pp. 723-732, 1984.

[17] F. A. McDonald, "On the precursor in laser-generated ultrasound waveforms in metals," Applied Physics Letters, vol. 56, no. 3, pp. 230-232, 1990.

[18] J. B. Spicer, A. D. W. McKie, and J. W. Wagner, "Quantitative theory for laser ultrasonic waves in a thin plate," Applied Physics Letters, vol. 57, no. 18, pp. 1882-1884, 1990.

[19] M. Dubois, F. Enguehard, L. Bertrand, M. Choquet, and J.P. Monchalin, "Modeling of laser thermoelastic generation of ultrasound in an orthotropic medium," Applied Physics Letters, vol. 64 , no. 5, pp. 554-556, 1994.

[20] M. A. Ezzat, A. S. El-Karamany, and M. A. Fayik, "Fractional ultrafast laser-induced thermo-elastic behavior in metal films," Journal of Thermal Stresses, vol. 35, no. 7, pp. 637-651, 2012.

[21] N. S. Al-Huniti and M. A. Al-Nimr, "Thermoelastic behavior of a composite slab under a rapid dual-phase-lag heating," Journal of Thermal Stresses, vol. 27, no. 7, pp. 607-623, 2004.

[22] J. K. Chen, J. E. Beraun, and C. L. Tham, "Comparison of onedimensional and two-dimensional axisymmetric approaches to the thermomechanical response caused by ultrashort laser heating," Journal of Optics A, vol. 4, no. 6, pp. 650-661, 2002.

[23] W.-S. Kim, L. G. Hector Jr., and R. B. Hetnarski, “Thermoelastic stresses in a bonded layer due to repetitively pulsed laser radiation," Acta Mechanica, vol. 125, no. 1-4, pp. 107-128, 1997.

[24] H. M. Youssef and A. A. El-Bary, "Thermoelastic material response due to laser pulse heating in context of four theorems of thermoelasticity," Journal of Thermal Stresses, vol. 37, no. 12, pp. 1379-1389, 2014.

[25] L. Yuan, K. Sun, Z. Shen, X. Ni, and J. Lu, "Theoretical study of the effect of enamel parameters on laser-induced surface acoustic waves in human incisor," International Journal of Thermophysics, vol. 36, no. 5-6, pp. 1057-1065, 2015.

[26] M. A. Elhagary, "A two-dimensional generalized thermoelastic diffusion problem for a thick plate subjected to thermal loading due to laser pulse," Journal of Thermal Stresses, vol. 37, no. 12, pp. 1416-1432, 2014.

[27] A. C. Eringen, Microcontinuum Field Theories. I. Foundations and Solids, Springer, New York, NY, USA, 1999.

[28] H. H. Sherief, F. A. Hamza, and H. A. Saleh, "The theory of generalized thermoelastic diffusion," International Journal of Engineering Science, vol. 42, no. 5-6, pp. 591-608, 2004.

[29] R. Kumar and T. Kansal, "Propagation of Lamb waves in transversely isotropic thermoelastic diffusive plate," International Journal of Solids and Structures, vol. 45, no. 22-23, pp. 58905913, 2008.
[30] H. M. Al-Qahtani and S. K. Datta, "Laser-generated thermoelastic waves in an anisotropic infinite plate: exact analysis," Journal of Thermal Stresses, vol. 31, no. 6, pp. 569-583, 2008.

[31] A. C. Eringen, "Plane waves in nonlocal micropolar elasticity", International Journal of Engineering Science, vol. 22, no. 8-10, pp. 1113-1121, 1984.

[32] R. S. Dhaliwal and A. Singh, Dynamical Coupled Thermoelasticity, Hindustan Publishers, New Delhi, India, 1980. 

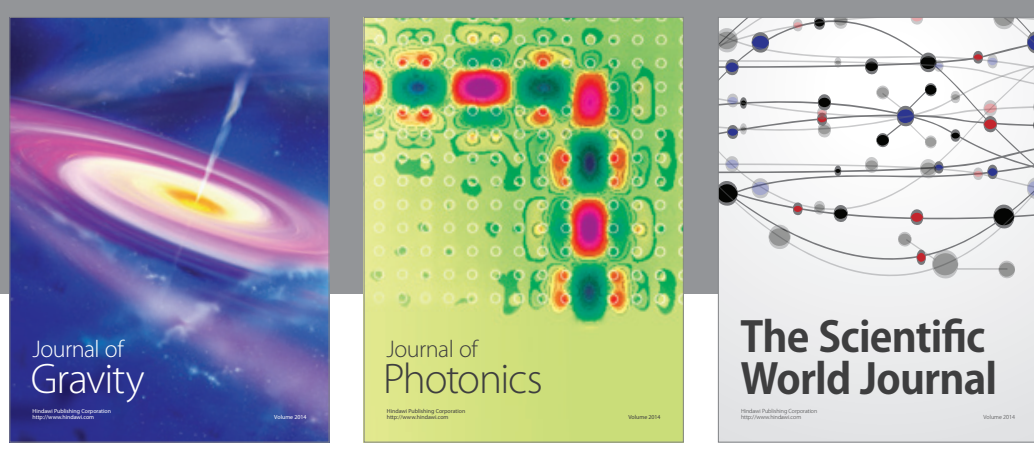

The Scientific World Journal
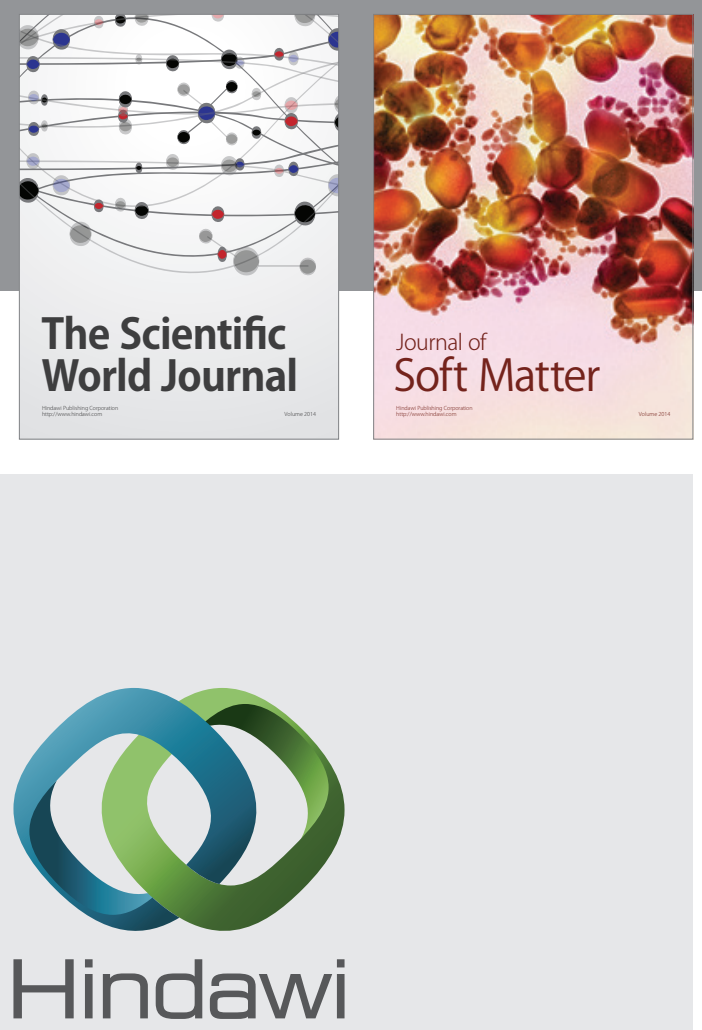

Submit your manuscripts at

http://www.hindawi.com

nternational Journal of

Statistical Mechanics
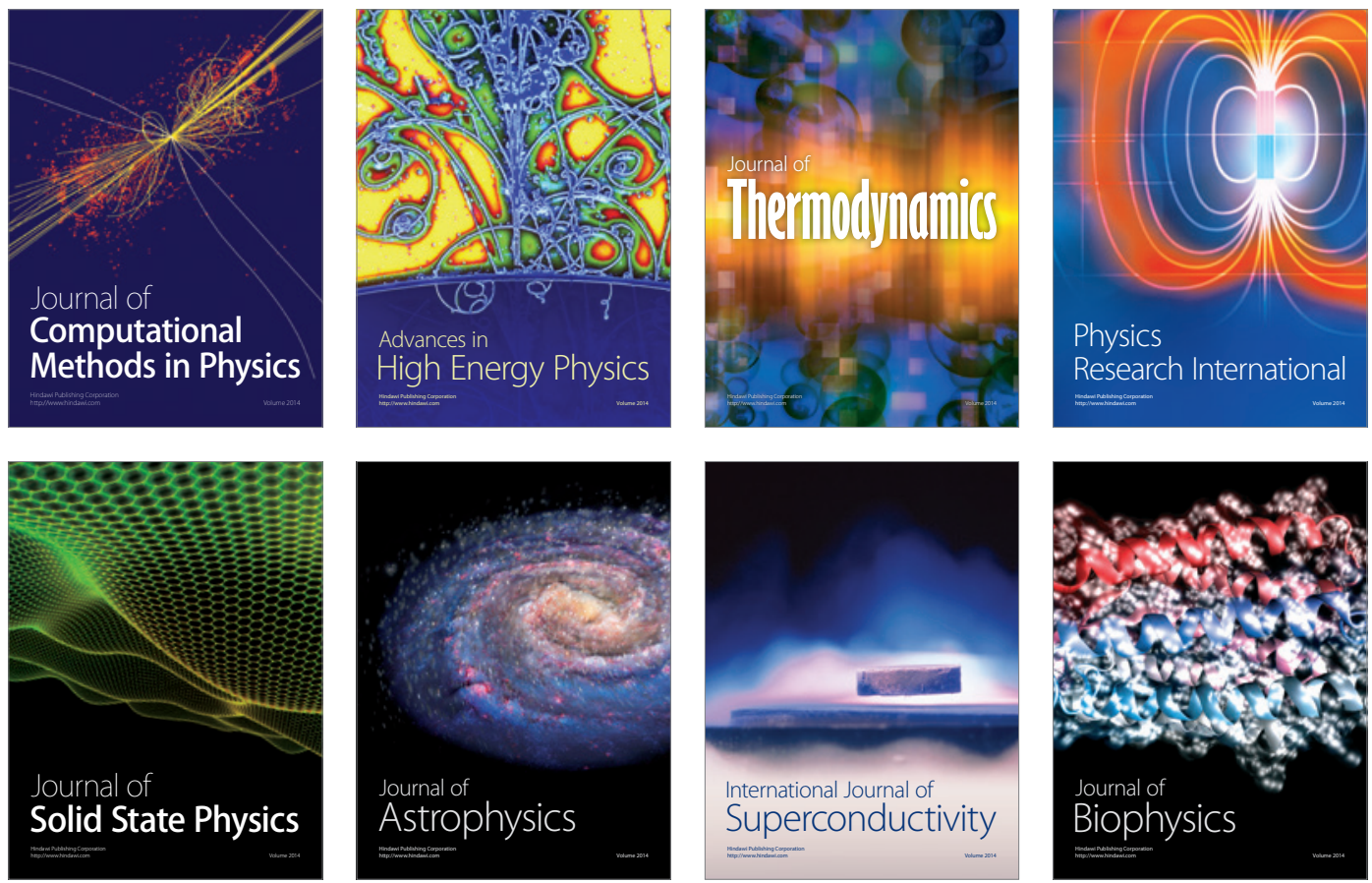
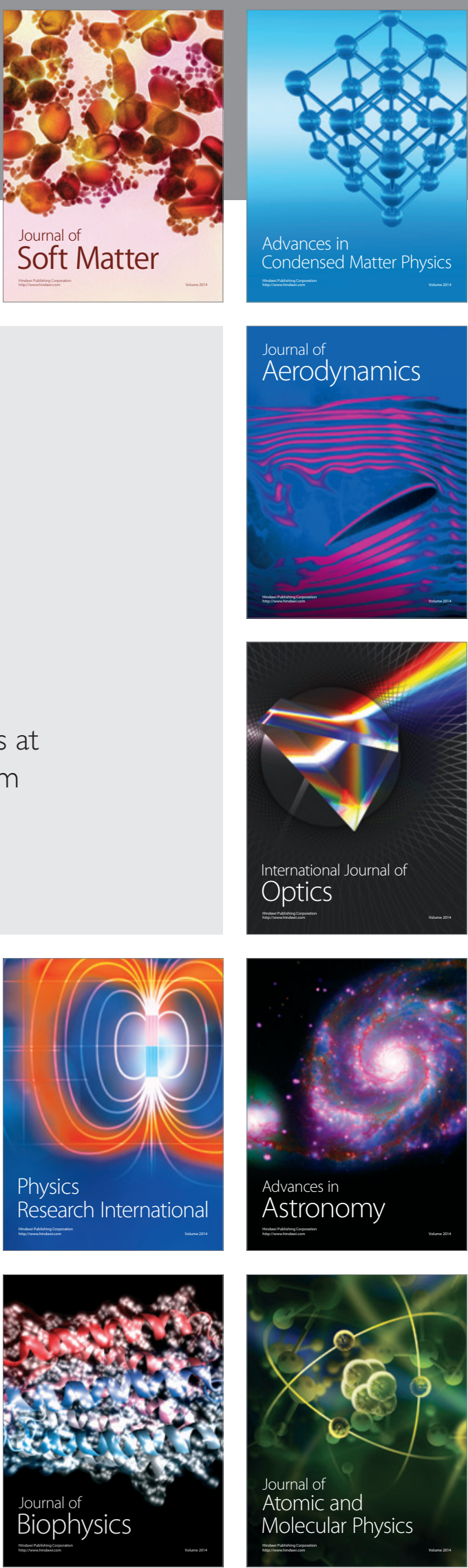